\title{
REPRESENTATIONS OF GRADED LIE ALGEBRAS
}

\author{
BY \\ LEONARD E. ROSS
}

Introduction. Let $L$ be a graded Lie algebra (see §2) over a commutative ring $R$ with unit. We shall deal with graded representations of $L$.

In $\$ 2$ we define the universal enveloping algebra $U$ of $L$ and prove a PoincaréBirkhoff-Witt theorem for $U$.

In $\$ 3$ we prove that if $L$ is a finite dimensional graded Lie algebra over a field of characteristic $\neq 2$, then $L$ has a faithful finite dimensional representation. A key lemma for this proof is a result on (nongraded) Lie algebras which states that if $L$ is a finite dimensional Lie algebra over a field of characteristic 0 then $L$ has a faithful, finite dimensional representation $f$ such that $f(x)$ is nilpotent whenever $\operatorname{ad} x$ is nilpotent.

This work is the first section of a doctoral thesis written under the direction of Professor Gerhard Hochschild. The author takes this opportunity to thank him for his generous advice and instruction.

1. The symmetric algebra built on a graded module. Let $\Gamma$ be an additive group, always assumed to have a subgroup $\Gamma_{0}$ of index 2 whose elements are called even. The elements of the complementary coset $\Gamma_{1}$ are called odd. If $\Gamma$ is the group of the integers, $\Gamma_{0}$ is necessarily the subgroup of the even integers.

For fixed $\Gamma, \Gamma_{0}$, define, for all $\gamma \in \Gamma$,

$$
1^{\gamma}=1,(-1)^{\gamma}=\left\{\begin{array}{cc}
-1, & \gamma \in \Gamma_{1} \\
1, & \gamma \in \Gamma_{0}
\end{array},(-1)^{\gamma \delta}=\left((-1)^{\gamma}\right)^{\delta}=\left((-1)^{\delta}\right)^{\gamma} .\right.
$$

Henceforth, " $R$ ", will denote a commutative ring with identity in which multiplication by 2 is an additive automorphism.

Let $L$ be a $\Gamma$-graded $R$-module. That is $L=\Sigma_{\gamma \in \Gamma} L_{\gamma}$, the $L_{\gamma}$ 's being $R$-submodules and the sum being direct. We say the elements of $L_{\gamma}$ are homogeneous of degree $\gamma$. The degree of a homogeneous element $x_{j}$ will sometimes be written as $d\left(x_{j}\right)$ or $d_{j}$. Written as $x_{\gamma}, \gamma \in \Gamma$, the element will always denote a homogeneous element of $L$.

If $\Gamma=(0,1)$ the homogeneous submodules will be written $L_{0}$ and $L_{1}$ and $L$ will be said to be semi-graded (SG). Generally, setting $L_{0}=\Sigma_{\gamma \text { even }} L_{\gamma}, L_{1}=\sum_{\gamma \text { odd }} L_{\gamma}$

Received by the editors October 22, 1964. 
we see that $L$ has a SG-structure called the $\Gamma_{0}$-associated semi-graded structure.

Let $T(L)$ be the tensor algebra over $R$ built on the module $L . T(L)=\sum_{i=0}^{\infty} T^{i}(L)$ $=R+L+L \otimes_{R} L+\cdots$. Let $S(L)=T(L) / J$, where $J$ is the two-sided ideal in $T(L)$ generated by the elements $x_{\lambda} \otimes x_{\mu}-(-1)^{\lambda \mu} x_{\mu} \otimes x_{\lambda} . S(L)$ is called the symmetric algebra in the graded sense based on the graded $R$-module $L$. If $L$ is $R$-free, $S(L)$ may be identified with the algebra of polynomials in the basis elements $\left(x_{\lambda}\right)$, with coefficients in $R$, and with relations $x_{\lambda} x_{\mu}=(-1)^{\lambda \mu} x_{\mu} x_{\lambda}$.

2. Graded Lie algebras and their enveloping algebras. Let $L$ be a graded Lie algebra (GLA) over the ring $R$. That is, $L$ is $\Gamma$-graded $R$-module, together with an $R$-bilinear multiplication [ ]: $L_{\lambda} \times L_{\mu} \rightarrow L_{\lambda+\mu}$ such that, for homogeneous $x_{\lambda}, x_{\mu}, x_{v}$ :

1. $\left[x_{\lambda}, x_{\mu}\right]=-(-1)^{\lambda \mu}\left[x_{\mu}, x_{\lambda}\right]$,

2a. $(-1)^{\lambda v}\left[x_{\lambda},\left[x_{\mu}, x_{v}\right]\right]+(-1)^{\lambda \mu}\left[x_{\mu},\left[x_{v}, x_{\lambda}\right]\right]+(-1)^{v \mu}\left[x_{v},\left[x_{\lambda}, x_{\mu}\right]\right]=0$,

2b. $\left[x_{\lambda},\left[x_{\lambda}, x_{\lambda}\right]\right]=0$.

If $\Gamma=(0,1)$, the $R$-submodules will be written as $L_{0}$ and $L_{1}$. We have $\left[L_{0}, L_{0}\right] \subset L_{0},\left[L_{0}, L_{1}\right] \subset L_{1},\left[L_{1}, L_{1}\right] \subset L_{0}$. Such an $L$ will be called a semigraded Lie algebra (SGLA). Generally, setting $L_{0}=\sum_{\gamma \in \Gamma_{0}} L_{\gamma}, L_{1}=\sum_{\gamma \in \Gamma_{1}} L_{\gamma}$, we see that $L$ has a SGLA structure, called the $\Gamma_{0}$-associated SGLA structure.

Let $U(L)=T(L) / I$, where $I$ is the two-sided ideal in $T(L)$ generated by the elements $x_{\lambda} \otimes x_{\mu}-(-1)^{\lambda \mu} x_{\mu} \otimes x_{\lambda}-\left[x_{\lambda}, x_{\mu}\right] . U(L)$ is called the universal enveloping algebra of $L$.

Let $M$ be a graded, unitary $R$-module and let $E^{*}(M)$ be the graded $R$-algebra of all graded $R$-endomorphisms of $M$. A graded $L$-module structure on $M$ is a linear map $\rho: L \rightarrow E^{*}(M)$, homogeneous of degree 0 , such that, for homogeneous $x_{\lambda}, x_{\mu}, \rho\left(\left[x_{\lambda}, x_{\mu}\right]\right)=\rho\left(x_{\lambda}\right) \rho\left(x_{\mu}\right)-(-1)^{\lambda \mu} \rho\left(x_{\mu}\right) \rho\left(x_{\lambda}\right)$. Extending $\rho$ to a unitary $R$-algebra homomorphism of $T(L)$ into $E^{*}(M)$, we have $\rho(I)=0$. Hence $\rho$ induces a unitary homomorphism of $U$ into $E^{*}(M)$, i.e., $M$ has the structure of a graded $U$-module over the graded algebra $U$, in which the grading is as follows. Let $u_{\lambda}$ be the image of $x_{\lambda}$ in $U(L)$. The grading on elements $u_{\lambda_{1}}^{e_{1}} \cdots u_{\lambda_{q}}^{e_{q}}$ is $\sum e_{j} \lambda_{j}$. We obtain in this fashion a 1-1 correspondence between graded $L$-module structures and unitary, graded $U$-module structures.

THEOREM 2.1. Suppose $L$ is homogeneously free over $R$ and let $\left(x_{j}\right)$ be a simply ordered, free, homogeneous $R$-basis of L. Let $u_{j}$ be the image of $x_{j}$ in $U$. Then 1 and the ordered monomials

2.1. $u_{j_{1}} \cdots u_{j_{n}}$, with $j_{1} \leqq \cdots \leqq j_{n}$, and $j_{k}<j_{k+1}$ whenever $d_{j_{k}}$ is odd, for $k=1, \cdots, n-1$,

form a free $R$-basis for $U$.

Proof. As in the nongraded case, the fact that these ordered monomials span $U$ is obtained from the "straightening" procedure. 
Let $U_{n}$ be the canonical image of $T_{n}$, where $T_{n}=\sum_{i \leqq n} T^{i}(L)$. The $U_{n}$ constitute an increasing filtration of the $R$-module $U . U_{0}$ is spanned by 1 . Assume $U_{n-1}$ is spanned by 1 and the ordered monomials $u_{j_{1}} \cdots u_{j_{n-1}}$. For the monomials $u_{j_{1}} \cdots u_{j_{n}}$ there are two cases to consider.

1. If $j_{r}>j_{r+1}$, for some $r$, then $u_{j_{1}} \cdots u_{j_{r}} u_{j_{r+1}} \cdots u_{j_{n}}=\varepsilon u_{j_{1}} \cdots u_{j_{r+1}} u_{j_{r}} \cdots u_{j}$. $+u_{j_{1}} \cdots\left[u_{j_{r}}, u_{j_{r+1}}\right] \cdots u_{j_{n}}$, with $\varepsilon= \pm 1$ and $u_{j_{1}} \cdots\left[u_{j_{r}}, u_{j_{r+1}}\right] \cdots u_{j_{n}} \in U_{n-1}$.

2. If $j_{r}=j_{r+1}$ with $d_{j_{r}}$ odd, then $u_{j_{1}} \cdots u_{j_{r}} u_{j_{r+1}} \cdots u_{j_{n}}=(1 / 2) u_{j_{1}} \cdots u_{j_{r-1}}\left[u_{j_{r}}, u_{j_{r}}\right]$ $\cdots u_{j_{n}} \in U_{n-1}$.

Hence, by induction on the number of inversions of order in $\left(j_{1}, \cdots, j_{n}\right)$ we show that the ordered monomials in 2.1 span $U_{n}$. First, if there are repetitions of indices $i_{r}$ in $\left(i_{1}, \cdots, i_{n}\right)$ with $d_{i_{r}}$ odd, then, by using 1 and 2 above, we see that $u_{i_{1}} \cdots u_{i_{n}} \in U_{n-1}$, whence by the induction hypothesis the monomial $u_{i_{1}} \cdots u_{i_{n}}$ is a sum of the ordered monomials in $U_{n-1}$. Assuming then no repetitions of indices corresponding to elements of odd degree we see that the theorem holds trivially when the number of inversions is zero. Assume the theorem is true for $p$ inversions. For $p+1$ inversions by using 1 on the first contiguous inversion that occurs reading from left to right, we have, by the induction hypothesis, that $u_{i_{1}} \cdots u_{i_{n}}$ is the sum of ordered monomials in $U_{n}$.

It remains to be shown that the ordered monomials are $R$-linearly independent. To prove this we define a graded $L$-module structure on $S(L)$, denoted by $(x, z) \rightarrow x \cdot z$, hence that of a graded $U(L)$-module structure on $S(L)$. With some modifications necessary to take the grading into account, the proof proceeds as in [1, Lemma 3.5, p.271].

The modifications are as follows. For $P=z_{i_{1}}^{e_{1}} \cdots z_{i_{p}}^{e_{p}} \in S(L)$, the degree of $P$ is $\sum e_{k} d_{i_{k}}$, and the height of $P$ is $\sum e_{k}$. Let $S^{\gamma}$ denote the subspace of $S(L)$ consisting of elements of degree $\gamma$, and let $S_{q}$ denote the subspace consisting of polynomials of height $\leqq q$. Then for each integer $p$ there is a unique homomorphism $L \otimes S_{p} \rightarrow S(L)$ such that

(0) $L_{\lambda} \cdot S^{\gamma} \subset S^{\lambda+\gamma}$

(1'a) $x_{j} \cdot z_{I}=z_{j} z_{I}, d_{j}$ even (odd), $j \leqq I(j \leqq I$ with $j \notin I)$,

(1'b) $x_{j} \cdot z_{I}=(1 / 2)\left[x_{j}, x_{j}\right] \cdot z_{J}, d_{j}$ odd, $j \leqq I$ with $I=(j, J)$,

(3) $x_{j} \cdot\left(x_{k} \cdot z_{J}\right)=(-1)^{\lambda \gamma^{\gamma}} x_{k} \cdot\left(x_{j} \cdot z_{J}\right)+\left[x_{j}, x_{k}\right] \cdot z_{J}, z_{J} \in S_{p-1}, \lambda=d\left(x_{j}\right)$, $\gamma=d\left(x_{k}\right)$.

Equations (2) and (4) of the above reference remain unchanged. At the inductive step it is easily seen that $x_{j} \cdot\left(x_{k} \cdot z_{I}\right)-(-1)^{\lambda \mu} x_{k} \cdot\left(x_{j} \cdot z_{I}\right)=\left[x_{j}, x_{k}\right] \cdot z_{I}$, where $\lambda=d\left(x_{j}\right), \mu=d\left(x_{k}\right)$, whenever $j, k \leqq I$ and $z_{I} \in S_{p}$. For if $d\left(x_{j}\right)$ is odd and $j=k$ with $j \notin I$ we have $2 x_{j} \cdot\left(x_{j} \cdot z_{I}\right)=2 x_{j} \cdot\left(z_{j} z_{I}\right)=\left[x_{j}, x_{j}\right] \cdot z_{I}$; whereas if $j \in I, I=(j, J)$, we have $2 x_{j} \cdot\left(x_{j} \cdot z_{I}\right)=2 x_{j} \cdot\left(x_{j} \cdot\left(z_{j} z_{J}\right)\right)=2 x_{j} \cdot\left((1 / 2)\left[x_{j}, x_{j}\right] \cdot z_{J}\right)$ $=\left[x_{j},\left[x_{j}, x_{j}\right]\right] \cdot z_{J}+\left[x_{j}, x_{j}\right] \cdot\left(x_{j} \cdot z_{J}\right)=\left[x_{j}, x_{j}\right] \cdot z_{I}$.

The other cases that arise under the condition that $j, k \leqq I$ and the remainder of the proof of the existence of the $L$-module structure are as in the reference with the necessary sign changes arising from (3). This proves Theorem 2.1. 
Now let $G^{n}$ be the $R$-module $U_{n} / U_{n-1}$, and let $G$ be the direct $R$-module sum of the $G^{n}$. $G$ has a multiplication induced by that of $U$ such that $G^{n} G^{m} \subset G^{n+m}$. $G$ is called the graded algebra associated to the filtered algebra $U$. Let $\psi_{n}$ be the canonical map of $T^{n}$ into $U_{n}, \Theta_{n}$ the canonical map of $U_{n}$ onto $G^{n}$, and let $\phi_{n}=\Theta_{n} \circ \psi_{n}$. Since $T^{n}$ is supplementary to $T_{n-1}$ in $T_{n}, \phi_{n}$ is surjective. The $\phi_{n}$ 's define an $R$-linear map $\phi$ of $T$ onto $G$.

Proposition 2.2. The map $\phi$ of $T$ onto $G$ is an epimorphism of algebras sending 1 into 1 , and $\phi$ annihilates the two-sided ideal generated by the elements $x_{\lambda} \otimes x_{\mu}-(-1)^{\lambda \mu} x_{\mu} \otimes x_{\lambda}$.

Proof. For $t \in T^{n}$ and $t^{\prime} \in T^{m}$, by definition of the multiplication in $G$, we have $\phi(t) \phi\left(t^{\prime}\right)=\phi\left(t t^{\prime}\right)$. Hence, $\phi$ is an algebra homomorphism, and it is clear that $\phi(1)=1$. Now for $x_{\lambda} \in L_{\lambda}, x_{\mu} \in L_{\mu}$, we have that $x_{\lambda} \otimes x_{\mu}-(-1)^{\lambda \mu} x_{\mu} \otimes x_{\lambda} \in T^{2}$ and the canonical image of this in $U_{2}$ is equal to that of $\left[x_{\lambda}, x_{\mu}\right]$, hence belongs to $U_{1}$. Hence $\phi\left(x_{\lambda} \otimes x_{\mu}-(-1)^{\lambda \mu} x_{\mu} \otimes x_{\lambda}\right)=0$. The above proposition shows that there is a unique epimorphism $\omega$ of the algebra $S(L)$ onto the algebra $G$ such that the following diagram is commutative:

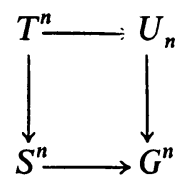

We have the following result.

COROLLARY. Under the hypothesis of Theorem 2.1 the above epimorphism $\omega$ is an isomorphism of $S(L)$ onto $G$.

Proof. It suffices to show that $\omega_{n}: S^{n}(L) \rightarrow G^{n}$ is injective. Since the monomials $x_{i_{1}}^{e_{1}} \cdots x_{i_{p}}^{e_{p}}$, with $i_{1}<\cdots<i_{p}, \sum e_{i}=n$, and $e_{k}=1$ if $d_{i_{k}}$ is odd, form a basis for $S(L)$, it will suffice to show that $\omega_{n}$ sends these elements onto $R$-linearly independent elements of $G^{n}$. Now $\omega_{n}\left(x_{i_{1}}^{e_{1}} \cdots x_{i_{p}}^{e_{p}}\right)$ is the coset $\bmod U_{n-1}$ of $u_{i_{1}}^{e_{1}} \cdots u_{i_{n}}^{e_{n}}$, and it is clear from Theorem 2.1 that these cosets are $R$-linearly independent elements of $G^{n}$.

\section{Representations of graded Lie algebras.}

Definition. Let $L$ be a GLA over a field $K$, and let $V$ be a vector space over $K$. A pre-representation of $L$ is a linear map $\rho: L \rightarrow E_{K}(V)$, such that $\rho\left[x_{\lambda}, x_{\mu}\right]$ $=\rho\left(x_{\lambda}\right) \rho\left(x_{\mu}\right)-(-1)^{\lambda \mu} \rho\left(x_{\mu}\right) \rho\left(x_{\lambda}\right)$. The term "representation" will always refer to a graded representation, that is, $V$ will be a graded $L$-module and $L_{\lambda}$ will send $V_{\mu}$ into $V_{\lambda+\mu}$.

Lemma 3.1. Let $A$ be a ring and let $B$ be a subring of $A$ such that $A$ is generated as a right $B$-module, with generators $\left(u_{i}\right)$. Then if $k$ is a left ideal in $B, k^{\prime}=\sum u_{i} k$ is a left ideal in $A$, 
Proof. Let $a \in A, h \in k^{\prime}$. Then $a=\sum u_{i} b_{i}$ with $b_{i}$ in $B$ and $h=\sum u_{i} k_{i}$, with $k_{i}$ in $k$. Hence $a h=\left(\sum_{j} u_{j} b_{j}\right)\left(\sum_{i} u_{i} k_{i}\right)=\sum_{i, j} u_{j} b_{j} u_{i} k_{i}$. But $u_{j} b_{j} u_{i}=\sum_{e} u_{e} b_{e i j}$, with $b_{e i j}$ in $B$. Hence $a h=\sum u_{e} b_{e i j} k_{i} \in k^{\prime}$.

Proposition 3.2. Let $L=L^{\prime} \oplus L^{\prime \prime}$ be a finite dimensional GLA over a field $K$ of characteristic $\neq 2$. Assume that $L^{\prime \prime}$ is a subalgebra of $L$ and that each homogeneous component of $L^{\prime}$ is of odd degree. Then every faithful, finite dimensional pre-representation (resp. representation) of $L^{\prime \prime}$ can be extended to a faithful, finite dimensional pre-representation (resp. representation) of $L$.

Proof. Let $\left(x_{i}\right)$ be a simply ordered, homogeneous $K$-basis of $L$ such that $x_{i}$, for $i=1, \cdots, m$ (resp. $i=m+1, \cdots, n$ ) constitutes a $K$-basis for $L^{\prime}$ (resp. $\left.L^{\prime \prime}\right)$. Let $u_{i}$ be the image of $x_{i}$ in $U(L)$ and let $V$ be the space spanned by $\left(u_{i_{1}} \cdots u_{i_{r}} \mid 1 \leqq i_{1}<\cdots<i_{r} \leqq m\right)$. Then, as a vector space, $U(L) \simeq U\left(L^{\prime \prime}\right) \oplus V U\left(L^{\prime \prime}\right)$. The pre-representation $\rho$ of $L^{\prime \prime}$, when extended to $U\left(L^{\prime \prime}\right)$, has a $U\left(L^{\prime \prime}\right)$-kernel $k$, such that $L \cap k=(0)$ in $U(L)$. If $\rho$ is a representation of $L^{\prime \prime}$, then $k$ is a homogeneous ideal in $U\left(L^{\prime \prime}\right)$.

Let $k^{\prime}=k+V k$. By Lemma 3.1, $k^{\prime}$ is a left ideal in $U(L)$ and is homogeneous whenever $k$ is homogeneous. Since $k$ is of finite co-dimension in $U\left(L^{\prime \prime}\right)$ and since $V$ is finite-dimensional and $U(L)=U\left(L^{\prime \prime}\right)+V U\left(L^{\prime \prime}\right), k^{\prime}$ is of finite co-dimension in $U(L)$. Finally, $L \cap k^{\prime}=(0)$; for let $y_{1} \in L^{\prime}, y_{2} \in L^{\prime \prime}, v \in V$ and $k_{1}, k_{2} \in k$ with $y_{1}+y_{2}=k_{2}+v k_{1}$, i.e. $\left(k_{2}-y_{2}\right)+\left(v k_{1}-y_{1}\right)=0$. Then the Birkhoff-Witt theorem (2.1) implies that $y_{2}=k_{2}$ and $y_{1}=v k_{1}$. Since $\rho$ is faithful, the first of these relations gives $y_{2}=0$. If $y_{1} \neq 0$ then the second relation gives $k_{1} \in K$ contrary to the definition of $k$. Hence also $y_{1}=0$, so that we have indeed $L \cap k^{\prime}=(0)$. Hence, by left multiplication in $U(L) / k^{\prime}, L$ has a faithful finite dimensional prerepresentation. If $k^{\prime}$ is homogeneous $U(L) / k^{\prime}$ has a gradation with which the prerepresentation is a representation.

COROLLARY. If L is a finite dimensional SGLA over a field of characteristic $\neq 2$, $L$ has a faithful, finite dimensional representation.

Proof. $L=L_{0}+L_{1}$, where $L_{0}$ is a Lie algebra. By Ado's theorem $L_{0}$ has a faithful, finite dimensional representation (of degree 0). Hence $L$ has a faithful finite dimensional representation.

LEMMA 3.3. Let $L$ be a finite dimensional Lie algebra over a field $F$ of characteristic 0 . Let $N$ be the maximal nilpotent ideal of $L$ and let $\rho$ be a finite dimensional representation of $L$ that is nilpotent on $N$. Let $x \in L$ and $\operatorname{suppose}$ ad $x$ is nilpotent. Then $\rho(x)$ is nilpotent.

Proof. Let $S$ be a maximal semi-simple subalgebra of $L$ and let $R$ be the radical of $L$. Then we have the semidirect sum decomposition $L=S+R$. Accordingly, if $x \in L$, write $x=s+r$, with $s$ in $S$ and $r$ in $R$. The endomorphism of $L / R$ induced 
by ad $x$ coincides with that induced by ads. Thus if $\operatorname{ad}_{L} x$ is nilpotent so is $\operatorname{ad}_{S} s$. It follows that every representation of $S$ maps $s$ onto a nilpotent linear endomorphism. In fact by [Theorem 17, p. 100 of [2]], there exists an $h \in S$ such that $[h, s]=s$. Then $F h+F s$ is a solvable subalgebra of $S$ whose derived algebra is $F s$. Now the assertion follows from Lie's theorem, which states that if $L$ is a solvable Lie algebra over a field of characteristic 0 , then every finite dimensional semisimple $L$-module is annihilated by $[L, L]$. In particular, $\operatorname{ad}_{L} S$ is nilpotent. It follows that $F s+N$ is nilpotent on $L$. Now $[\operatorname{ad}(s+r), \operatorname{ad} s]=\operatorname{ad}[r, s]$, and $[r, s] \in N$. Hence $F(s+r)+F s+N$ is nilpotent on $L$. Therefore ad $r$ is nilpotent on $L$. Hence $r \in N$. Now $\rho(s)$ is nilpotent, $\rho(N)$ is nilpotent and $[\rho(s), \rho(N)] \subset \rho(N)$, so that $F \rho(s)+\rho(N)$ is nilpotent. Hence $\rho(x)$ is nilpotent.

Proposition 3.4. Let $L$ be a finite dimensional, evenly graded Lie algebra over a field of characteristic 0 . Then $L$ has a faithful, finite dimensional representation.

Proof. By Lemma 3.3 and Ado's theorem, $L$ has a faithful, finite dimensional pre-representation $\rho^{*}$, such that $\rho^{*}(x)$ is nilpotent whenever ad $x$ is nilpotent. Let $V$ be the representation space of $\rho^{*}$. For each $\gamma \in \Gamma$, let $V_{\gamma}$ be a copy of $V$ and let $\pi_{\gamma}$ be an isomorphism of $V_{\gamma}$ onto $V$. Let $V_{0}=V$ and let $\pi_{0}$ be the identity map on $V$. For $x \in L_{\lambda}$ and $v \in V_{\gamma}$ define

$$
\rho(x)(v)=\pi_{\lambda+\gamma}^{-1}\left(\rho^{*}(x)\left(\pi_{\gamma}(v)\right)\right) .
$$

One verifies directly that $\rho$ is a representation of $L$ on $\sum_{\gamma \in \Gamma} V_{\gamma}$. Moreover, if $V^{\prime}$ is the submodule of $\sum_{\gamma \in \Gamma} V_{\gamma}$ that is generated by $V$, then $\rho$ induces a faithful representation of $L$ on $V^{\prime}$. Hence it suffices to show that $V^{\prime}$ is finite dimensional. Note that if $x \in L_{\gamma}$ and ad $x$ is not nilpotent then $\gamma$ must be of finite order, because $L$ is finite dimensional. Also the set of $\gamma$ 's for which such an $x$ exists must be finite. Hence the group generated by these $\gamma$ 's is a finite subgroup $\Delta$ of $\Gamma$.

Now we choose an ordered, homogeneous basis $x_{1}, \cdots, x_{r}, y_{1}, \cdots, y_{s}$ of $L$ such that each ad $x_{i}$ is nilpotent and each $y_{j}$ lies in some $L_{\gamma}$ with $\gamma \in \Delta$. Each $\rho^{*}\left(x_{i}\right)$ is nilpotent, so that there is a non-negative integer $q$ such that $\rho^{*}\left(x_{i}\right)^{q}=0$, for all $i$. If $v \in V_{\gamma}$ and $x_{i} \in L_{\lambda}$ we have $\rho\left(x_{i}\right)^{k}(v)=\pi_{k \lambda+\gamma}^{-1}\left(\rho^{*}\left(x_{i}\right)^{k}\left(\pi_{\gamma}\left(v_{\gamma}\right)\right)\right)$, whence $\rho\left(x_{i}\right)^{q}=0$.

Now $V^{\prime}$ consists of all linear combinations $\rho\left(x_{1}\right)^{e_{1}} \cdots \rho\left(x_{r}\right)^{e_{r}} \rho\left(y_{1}\right)^{f_{1}} \cdots \rho\left(y_{s}\right)^{f_{s}}(v)$, with $v \in V$. These are 0 if one of the $e_{i}$ 's is $\geqq q$. Hence it suffices to take $0 \leqq e_{i}<q$. The elements $\rho\left(y_{1}\right)^{f_{1}} \cdots \rho\left(y_{s}\right)^{f_{s}}(v)$ lie in the finite dimensional space $\sum_{\gamma \in \Delta} V_{\gamma}$. Hence it is clear that $V^{\prime}$ is finite dimensional.

COROLLARY. If L is a finite dimensional GLA over a field of characteristic 0 , then $L$ has a faithful, finite dimensional representation.

Proof. $L=\sum_{\gamma \text { even }} L_{\gamma}+\sum_{\gamma \text { odd }} L_{\gamma}$. By Proposition $3.4, \sum_{\gamma \text { even }} L_{\gamma}$ has a faithful, finite dimensional representation which, by Proposition 3.2 may be extended to a faithful, finite dimensional representation. 
Proposition 3.5. Let $L$ be a finite dimensional GLA over a field of characteristic $\neq 2$. Then $L$ has a faithful finite dimensional representation.

Proof. $L=\sum_{\gamma \text { even }} L_{\gamma}+\sum_{\gamma \text { odd }} L_{\gamma}$. By Proposition 3.2 it suffices to show that $L^{\prime}=\Sigma_{\gamma \text { even }} L_{\gamma}$ has a faithful, finite dimensional representation.

Let $\left(x_{1}, \cdots, x_{n}\right)$ be an ordered, homogeneous basis for $L^{\prime}$ such that $\operatorname{ad}_{L^{\prime}} x_{i}$ is nilpotent, for $i=1, \cdots, r$, and $x_{i}$, for $i=r+1, \cdots, n$, lies in some $L_{\gamma}$ with $\gamma$ of finite order. For $i=1, \cdots, r$ there is a $p$-polynomial $m_{i}(\lambda)=\lambda^{p^{m_{i}}}$ such that $z_{i}=x_{i}^{p^{m_{i}}}$ is in the center of $U\left(L^{\prime}\right)$. For $i=r+1, \cdots, n$ there is a $p$-polynomial $m_{i}(\lambda)=$ $\lambda^{p^{m_{i}}}+a_{1} \lambda^{p^{m_{i}-1}}+\cdots+a_{m_{i}} \lambda$ such that $z_{i}=x_{i}^{p^{m_{i}}}+a_{1} x_{i}^{p^{m_{i}-1}}+\cdots+a_{m_{i}} x_{i}$ is in the center of $U\left(L^{\prime}\right)$.

Now $U\left(L^{\prime}\right)$ is graded, and the center of $U\left(L^{\prime}\right)$ is homogeneous. Hence, by dropping some terms of exponent $<p^{m_{i}}$ if necessary, we may assume $z_{i}$ is homogeneous. Hence, by Lemma 5.4 of [2], the elements $z_{1}^{h_{1}} \cdots z_{n}^{h_{n}} u_{1}^{\lambda_{1}} \cdots u_{n}^{\lambda_{n}}, h_{i} \geqq 0$, $0 \leqq \lambda_{i}<p^{m_{i}}$, form a basis for $U\left(L^{\prime}\right)$. Let $B$ be the ideal in $U$ generated by the $z_{i}$. Then $B$ is homogeneous since each generator is homogeneous. As in the proof of Theorem 5.11 of [2] the cosets of the elements $u_{1}^{\lambda_{1}} \cdots u_{n}^{\lambda_{n}}, 0 \leqq \lambda_{i}<p^{m_{i}}$ form a basis for $U / B$. Hence this algebra is a finite dimensional graded algebra and the canonical mapping $a \rightarrow \bar{a}, a \in L^{\prime}$, is a monomorphism of $L^{\prime}$ into $\bar{U}=U / B$. It follows that $L^{\prime}$ has a faithful, finite dimensional representation.

\section{BIBLIOGRAPHY}

1. H. Cartan and S. Eilenberg, Homological algebra, Princeton Univ. Press, Princeton, N. J., 1956.

2. N. Jacobson, Lie algebras, Interscience, New York, 1962.

UNIVERSITY OF CALIFORNIA, Los Angeles, California 